\title{
Runaway Positrons in Fusion Plasmas
}

\author{
T. Fülöp and G. Papp \\ Department of Applied Physics, Nuclear Engineering, Chalmers University of Technology and Euratom-VR Association, \\ Göteborg, Sweden
}

(Received 28 December 2011; revised manuscript received 13 May 2012; published 29 May 2012)

\begin{abstract}
Runaway positrons can be produced in the presence of runaway electron avalanches in magnetized plasmas. In this Letter, we determine the positron distribution, the fraction of runaway positrons, and the parametric dependences of their synchrotron radiation spectrum. We show that the maximum production occurs around $\gamma_{e} \simeq 30$, where $\gamma_{e}$ is the Lorentz factor of the fast electrons. For an avalanching positron distribution typical of tokamak plasmas, the maximum of the synchrotron radiation spectrum should be around a micron. The radiated power and spectrum shape are sensitive to the plasma parameters. Apart from its intrinsic interest, detection of radiation from positrons could be a diagnostic tool to understand the properties of the medium they propagate through.
\end{abstract}

DOI: 10.1103/PhysRevLett.108.225003

PACS numbers: 52.27.Ny, 41.75.Ht, 52.25.Os, 52.55.Fa

Introduction.-Relativistic electron populations originating from runaway electron avalanches have been frequently observed in various plasmas, e.g., large tokamak disruptions [1] and electric discharges associated with thunderstorms [2]. The energetic electrons produced in the avalanches may give rise to electron-positron pair production. Pair production can occur in collisions between runaway electrons and thermal ions if the runaway energy exceeds three times the electron rest mass. In electric discharges associated with thunderstorms, electrons can be accelerated up to $100 \mathrm{MeV}$ [2]. In postdisruption plasmas in large tokamaks, the energy of the runaway electrons is in the $10-20 \mathrm{MeV}$ range. Therefore, in these plasmas the typical runaway energy is well above the threshold for pair production, and positrons should therefore be present in large quantities [3]. The positrons generated by runaway electron avalanches are already highly relativistic at birth; in addition, they experience acceleration by the electric field. The aim of the present work is to determine the distribution of positrons at birth, their subsequent fate, and the synchrotron radiation emitted by them. The production rate is calculated by using a pair-production cross section valid for arbitrary energies and a runaway electron distribution typical for avalanching. To obtain the positron velocity distribution, the Fokker-Planck equation including the positron production and annihilation rates and slowingdown terms is solved. The result is used to calculate the fraction of runaway positrons and the parametric dependences of their synchrotron radiation spectrum.

Positron production.-The cross section for the production of electron-positron pairs by electrons in the field of a nucleus is calculated numerically in Ref. [4] for incident particle energies from the threshold to $100 \mathrm{MeV}$. A fit to the numerical results over the entire energy range is

$$
\sigma_{\text {tot }}=a Z^{2} \ln ^{3}\left(\frac{\gamma_{e}+x_{0}}{3+x_{0}}\right)
$$

where $a=5.22 \mu b\left(1 b=10^{-28} \mathrm{~m}^{2}\right), x_{0}=3.6, Z$ is the charge of the stationary particle, and $\gamma_{e}$ is the Lorentz factor of a fast electron. For high energies Eq. (1) agrees with the ultrarelativistic limit of the cross section for pair production $\sigma_{\gamma \gg 1} \simeq\left[28\left(Z \alpha r_{e}\right)^{2} / 27 \pi\right] \ln ^{3} \gamma_{e}$ [5], where $\alpha \simeq 1 / 137$ is the fine-structure constant, and $r_{e}$ is the classical electron radius. The ultrarelativistic approximation $\sigma_{\gamma \gg 1}$ describes well the behavior of the cross section for incident electron energies above $150 m_{e} c^{2}$, where $m_{e}$ is the electron rest mass. Near the threshold, Eq. (1) agrees with the expression for the threshold total cross section, which is $\sigma_{\text {th }}=0.013 Z^{2}\left(\gamma_{e}-3\right)^{3} \mu b$ [4].

Fig. 1 shows the total cross section from Eq. (1) as a function of incident electron energy, together with the ultrarelativistic approximation $\sigma_{\gamma \gg 1}$, the threshold approximation $\sigma_{\text {th }}$, and the annihilation cross section. The threshold formula is reasonably correct for energies below

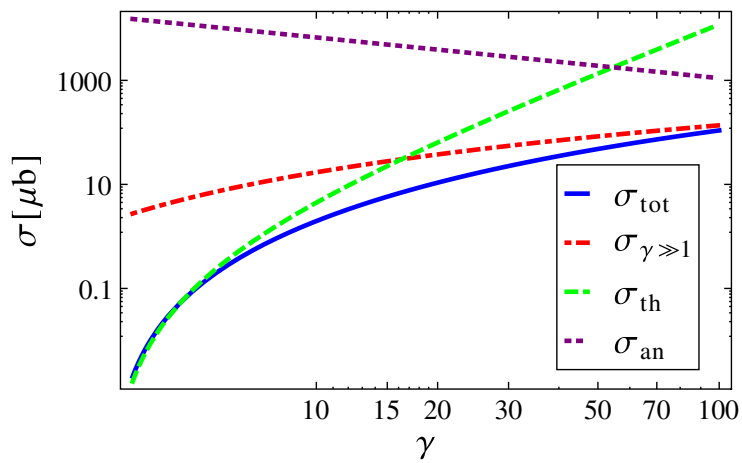

FIG. 1 (color online). Total cross section in $\mu b$ for $Z=1$, as a function of the electron Lorentz factor. The dash-dotted line is the ultrarelativistic approximation $\sigma_{\gamma \gg 1}$, the dashed line is the threshold approximation $\sigma_{\text {th }}$, and the solid line is the numerically calculated cross section (1). The annihilation cross section (2) is a function of the positron Lorentz factor and is shown by a dotted line. 
$5 m_{e} c^{2}$. Since the runaway electrons typically have energies in the range 5 to $70 m_{e} c^{2}, \sigma_{\text {tot }}$ should be used.

Since the positron creation cross section increases rapidly from a threshold, the positrons will be born with Lorentz factors $\gamma_{+} \gg 1$. The process of annihilation is about two orders of $1 / \alpha$ more probable than that of creating positrons. The dotted line in Fig. 1 shows the annihilation cross section,

$$
\begin{aligned}
\sigma_{a n}= & \frac{\pi r_{e}^{2}}{1+\gamma_{+}}\left[\frac{\gamma_{+}^{2}+4 \gamma_{+}+1}{\gamma_{+}^{2}-1} \ln \left(\gamma_{+}+\sqrt{\gamma_{+}^{2}-1}\right)\right. \\
& \left.-\frac{\gamma_{+}+3}{\sqrt{\gamma_{+}^{2}-1}}\right] \frac{2 \pi \alpha / p_{+}}{1-e^{-2 \pi \alpha / p_{+}}} .
\end{aligned}
$$

The positron production rate $d n_{\text {prod }}^{+} / d t \equiv S_{p}$ can be written in a general form as $S_{p}=n_{i} \int_{p>p_{\text {min }}} f_{e}^{\mathrm{RE}} \sigma_{\text {tot }} v_{e} d^{3} p_{e}=$ $2 \pi n_{i} \int_{p>p_{\min }} f_{e}^{\mathrm{RE}} \sigma_{\mathrm{tot}} v_{e} p_{e \perp} d p_{e \perp} d p_{e \|}$, where $n_{i}$ is the number density of the ions, $\sigma_{\text {tot }}$ is given in Eq. (1), and the threshold momentum is $p_{\min }=3$. Here $f_{e}^{\mathrm{RE}}$ is the runaway electron distribution function, $p_{e}=\gamma_{e} v_{e} / c$ is the normalized relativistic momentum, $c$ is the speed of light, and $\|$ and $\perp$ are parallel and perpendicular directions with respect to the magnetic field.

If the normalized parallel electric field $E=$ $e\left|E_{\|}\right| \tau / m_{e} c \gg 1$, the runaway tail has the character of a beam, so the parallel momentum is much larger than the perpendicular, $p_{e \perp} \ll p_{e \|} \simeq p_{e}$. Here, $\tau=1 / 4 \pi r_{e}^{2} n_{e} c \ln \Lambda$ is the collision time for relativistic positrons and electrons and $\ln \Lambda$ is the Coulomb logarithm. If $E \gg 1$, most of the runaway electrons are produced by avalanching, in which case the runaway electron density $n_{r}$ increases according to $d n_{r} / d t \simeq n_{r}(E-1) / c_{z} \tau \ln \Lambda$ [6], where $c_{z}=\sqrt{3\left(Z_{\text {eff }}+5\right) / \pi}$ and $Z_{\text {eff }}$ is the effective ion charge. Then the distribution function of relativistic runaway electrons is

$f_{e}^{\mathrm{RE}}\left(p_{e \|}, p_{e \perp}\right)=\frac{n_{r} \hat{E}}{2 \pi c_{z} p_{e \|} \ln \Lambda} \exp \left(-\frac{p_{e \|}}{c_{z} \ln \Lambda}-\frac{\hat{E} p_{e \perp}^{2}}{2 p_{e \|}}\right)$,

where $\hat{E}=(E-1) /\left(1+Z_{\text {eff }}\right)$ [7]. In the limit of $p_{e \|} \simeq$ $p_{e} \gg 1$, we can approximately write $p_{e} \simeq \gamma_{e}$. To illustrate how many positrons are generated as a function of runaway electron energy, the differential production rate can be calculated by using the runaway electron distribution from Eq. (3) and evaluating the integral over $p_{e \perp}$ in the expression for $S_{p}$,

$$
\frac{d S_{p}}{d p_{e}} \simeq \frac{n_{i} n_{r} c}{c_{z} \ln \Lambda} \exp \left(-\frac{p_{e}}{c_{z} \ln \Lambda}\right) \sigma_{\text {tot }}
$$

$d S_{p} / d p_{e}$ is the number of positrons produced per second per unit volume by the electrons with momentum $p_{e}$ corresponding to $\gamma_{e}=E_{e} / m_{e} c^{2}$. Note that although the distribution function in Eq. (3) is derived for toroidally symmetric plasmas, runaway electron distributions are generally expected to be beamlike in any circumstances, so the results should be qualitatively similar in other plasmas.
The differential positron production rate from Eq. (4) as function of runaway energy is shown in Fig. 2. For $\ln \Lambda=$ 10 , the maximum production occurs around $\gamma_{e} \simeq 30$ and the ultrarelativistic cross section used in Ref. [3] overestimates the positron production. As an example, the number of runaway electrons in a postdisruption plasma with a runaway current $I_{r}$ is $N_{r}=2 \pi R I_{r} / e c$, where $R$ is the major radius of the torus. For $R=3 \mathrm{~m}$ and $I_{r}=1 \mathrm{MA}$, we have $N_{r}=4 \times 10^{17}$, and we assume that the runaways are concentrated in a beam of total volume $1 \mathrm{~m}^{3}$, then the runaway density is $n_{r}=4 \times 10^{17} \mathrm{~m}^{-3}$. Taking into account collisions only between runaway electrons and hydrogenic ions with density $n_{i}=5 \times 10^{19} \mathrm{~m}^{-3}$, we find that the production rate is $S_{p}=\left(n_{r} n_{i} c / c_{z} \ln \Lambda\right) \times$ $\int_{3}^{\infty} e^{-p / c_{z} \ln \Lambda} \sigma_{\text {tot }} d p \simeq 1.5 \times 10^{13} \mathrm{~s}^{-1} \mathrm{~m}^{-3}$ (for $\ln \Lambda=10$ and $Z_{\text {eff }}=1.6$ ). Also, collisions with thermal electrons and impurities contribute to the number of positrons created. The number of positrons created in collisions with electrons is about the same order of magnitude as that from collisions with hydrogenic ions (although the threshold momentum is higher, most of the runaway electrons typically do exceed that as well). $S_{p}$ should therefore be multiplied by $M_{p} \equiv 1+n_{e} / n_{i}+\sum_{z} n_{z} Z^{2} / n_{i}$, where the summation is over all impurity species (regardless of whether they are fully ionized or not). Due to the substantial amount of high- $Z$ impurities present in the postdisruptive plasmas, this multiplicative factor can be several orders of magnitude. During a tokamak disruption, it has been estimated that at least $1 \mathrm{~g}$ of carbon can be released from the wall and reach the plasma center in less than 1 millisecond [9]. This amount of carbon, if it is distributed uniformly in a volume of about $80 \mathrm{~m}^{3}$, would correspond to a multiplicative factor of $M_{p} \simeq 450$. Note that $M_{p}$ can be large even if $Z_{\text {eff }}$ is order unity, because the expression for $M_{p}$ contains the full nuclear charge.

Source of positrons.-The production rate given in Eq. (4) only gives the number of positrons generated for

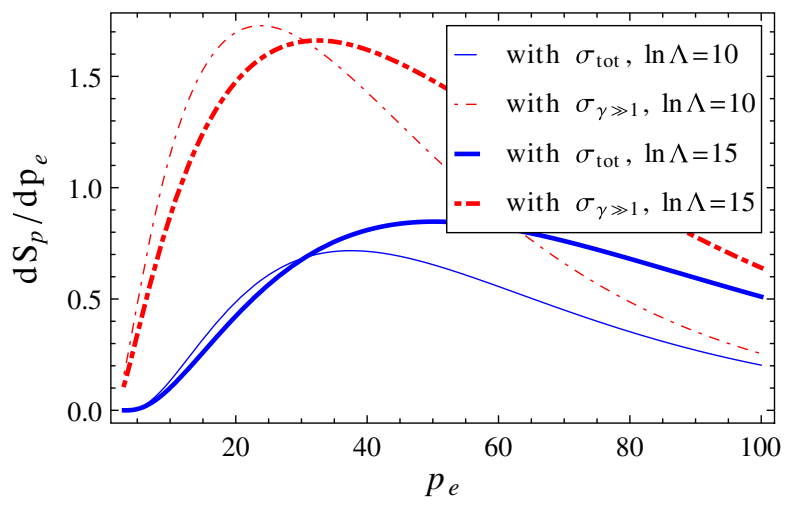

FIG. 2 (color online). Differential production rate normalized to $n_{i} n_{r} c / c_{z}$ as function of runaway energy from (4) using the runaway distribution from (3). The dash-dotted red line uses the ultrarelativistic approximation of the cross section $\sigma_{\gamma \gg 1}$, and the solid line the numerically calculated cross section (1). Thin lines are for $\ln \Lambda=10$ and thick lines are for $\ln \Lambda=15$. 
a certain incident electron energy and says nothing about their energy. If the probability distribution of positrons with momentum $p_{+}$generated from electrons of momentum $p_{e}, \mathcal{F}\left(p_{e}, p_{+}\right)$, is known, the positron velocity distribution can be calculated by using $s_{p} \equiv d f_{+} / d t=$ $n_{i} \int f_{e}^{\mathrm{RE}} \sigma_{\mathrm{tot}} v_{e} \mathcal{F}\left(p_{e}, p_{+}\right) d p_{e}$ as the source term in the kinetic equation. The production rate $S_{p}$ defined in Eq. (4) is the integral of the quantity we need to find $d n_{+} / d t=S_{p}=\int\left(d f_{+} / d t\right) d^{3} p_{+}$.

The mean energy of the positrons generated by an incoming electron with momentum $p_{e},\left\langle E_{+}\right\rangle\left(p_{e}\right)=$ $\int E_{+}\left(p_{+}\right) \mathcal{F}\left(p_{e}, p_{+}\right) d p_{+}$, has been calculated numerically in Ref. [4] and can be written as

$$
\frac{\left\langle E_{+}\right\rangle}{E_{e}}=\frac{1}{3}-0.0565 \ln \frac{E_{e}}{3 m_{e} c^{2}} .
$$

Here, the constants $(1 / 3,0.0565)$ are fitted parameters. The evaluation of the integral in $s_{p}$ is complicated by the fact that the relation of $E_{e}\left(E_{+}\right)$cannot be given in a closed form for Eq. (5). Therefore, we fitted the data given in Ref. [4] with a function that allows us to give $E_{+}\left(E_{e}\right)$ in an invertible form. The new fit has the form $E_{+}=0.44 E_{e}^{0.69}$ (energies in $\mathrm{MeV}$ ) and is slightly better in the high-energy region. The inverse can be obtained by inverting $E_{+}\left(E_{e}\right)$ or fitting, and the result is $E_{e}=3.28 E_{+}^{1.445}$. From this we can calculate the relation between the electron and positron momenta by using $E / m_{e} c^{2}=\sqrt{1+p^{2}}$. When the electron and positron momenta are large $p^{2} \gg 1$, this leads to $p_{e}=$ $4.42 p_{+}^{1.445}$

A simple estimate for the positron source can be found by solving the integral in $s_{p}$ assuming that $\mathcal{F}\left(p_{e}, p_{+}\right)$is a delta function at a point when Eq. (5) is satisfied, so that

$$
s_{p}^{\delta}=\int f_{e}^{\mathrm{RE}} \sigma_{\mathrm{tot}} v_{e} \delta\left(p_{e}-4.42 p_{+}^{1.445}\right) 4 \pi p_{e}^{2} d p_{e},
$$

where $p_{+}=\gamma_{+} v_{+} / c$ is the normalized momentum of the positrons. We have verified that the source term $s_{p}$ will be similar even if we model the probability $\mathcal{F}\left(p_{e}, p_{+}\right)$with a Maxwellian instead of a delta function.

Positron distribution.-In the absence of an electric field, a positron usually slows down initially due to synchrotron radiation emission, and when it has reached mildly relativistic energies collisions take over. For the energies of interest $\left(\gamma_{+} \lesssim 50\right)$ collisional slowing-down dominates, and the positron distribution function can be calculated from the kinetic equation

$\frac{\partial f_{+}}{\partial t}=\frac{1}{\tau p_{+}^{2}} \frac{\partial}{\partial p_{+}}\left[\left(1+p_{+}^{2}\right) f_{+}\right]-n_{e} v_{+} \sigma_{a n} f_{+}+s_{p}\left(p_{+}\right)$,

where the first term on the right is slowing down, the second is annihilation, and the last is the production rate.

Substituting the positron production rate from Eq. (6) into Eq. (7) allows us to determine the steady-state positron distribution. Figure 3(a) shows the numerical solution of Eq. (7), with $s_{p}$ from Eq. (6) and $\sigma_{a n}$ from Eq. (2). It shows that most positrons that survive the slowing-down without annihilation will have momentum below $p_{+}=10$ (corresponding to energies less than $5 \mathrm{MeV}$ ). The total number of positrons in the above-mentioned example (for $S_{p}=$ $1.5 \times 10^{13} \mathrm{~s}^{-1} \mathrm{~m}^{-3}$ and $\left.\tau=0.067 \mathrm{~s}\right)$ is $n_{+}=8 \times 10^{12} \mathrm{~m}^{-3}$. Taking into account also the multiplicative factor $M_{p}$, the number of positrons should be larger than in the case of positrons created by intense laser-solid interactions [8]; however, also the volume of the magnetic confinement device is also larger, and therefore detection is more challenging. The lifetime of a positron can be estimated from the annihilation cross section $\tau_{p}=1 / n_{e} v_{+} \sigma_{a n}$, and it is expected to be of the order of seconds.

If the electric field is strong enough, the positrons will run away in the opposite direction to the electrons and will acquire velocities close to the speed of light. In Eq. (7) the influence of the electric field on the distribution function has been neglected. We can nevertheless estimate the number of positrons that run away by investigating how many positrons have velocities above the critical velocity $v_{c}=c / \sqrt{2 E}$. When the electric field is neglected, the distribution function is isotropic in velocity space, whereas a tail is pulled out in the direction of the magnetic field if the electric field is retained. Interestingly, the neglect of the
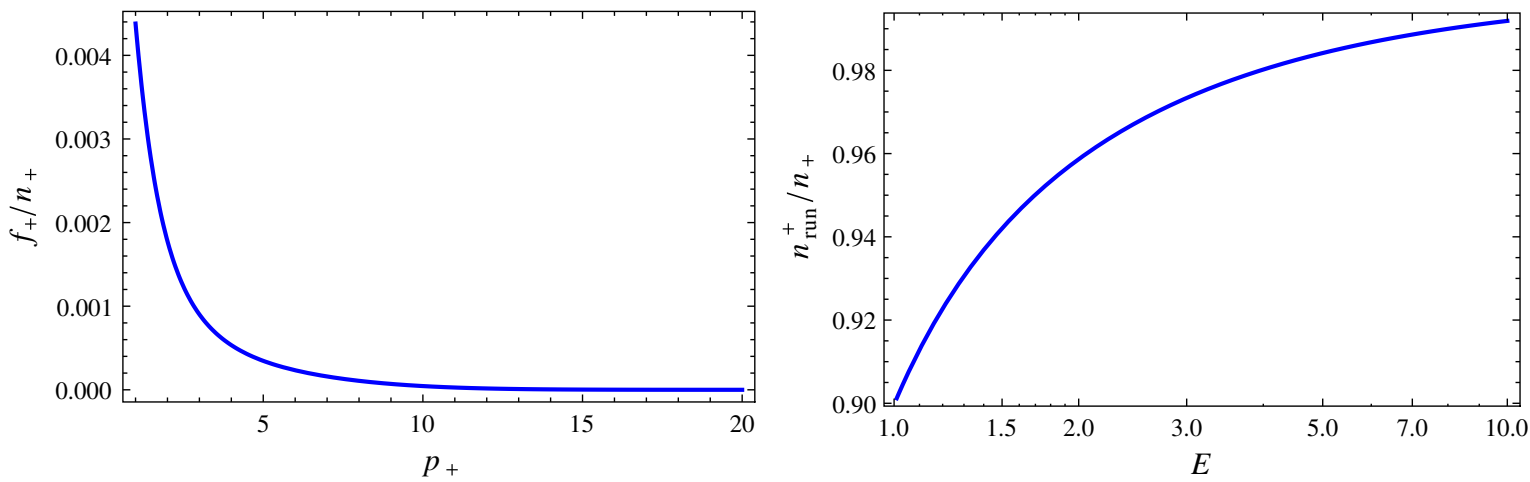

FIG. 3 (color online). (a) Normalized positron distribution as a function of normalized momentum. (b) Fraction of positrons that run away as a function of the normalized electric field. 

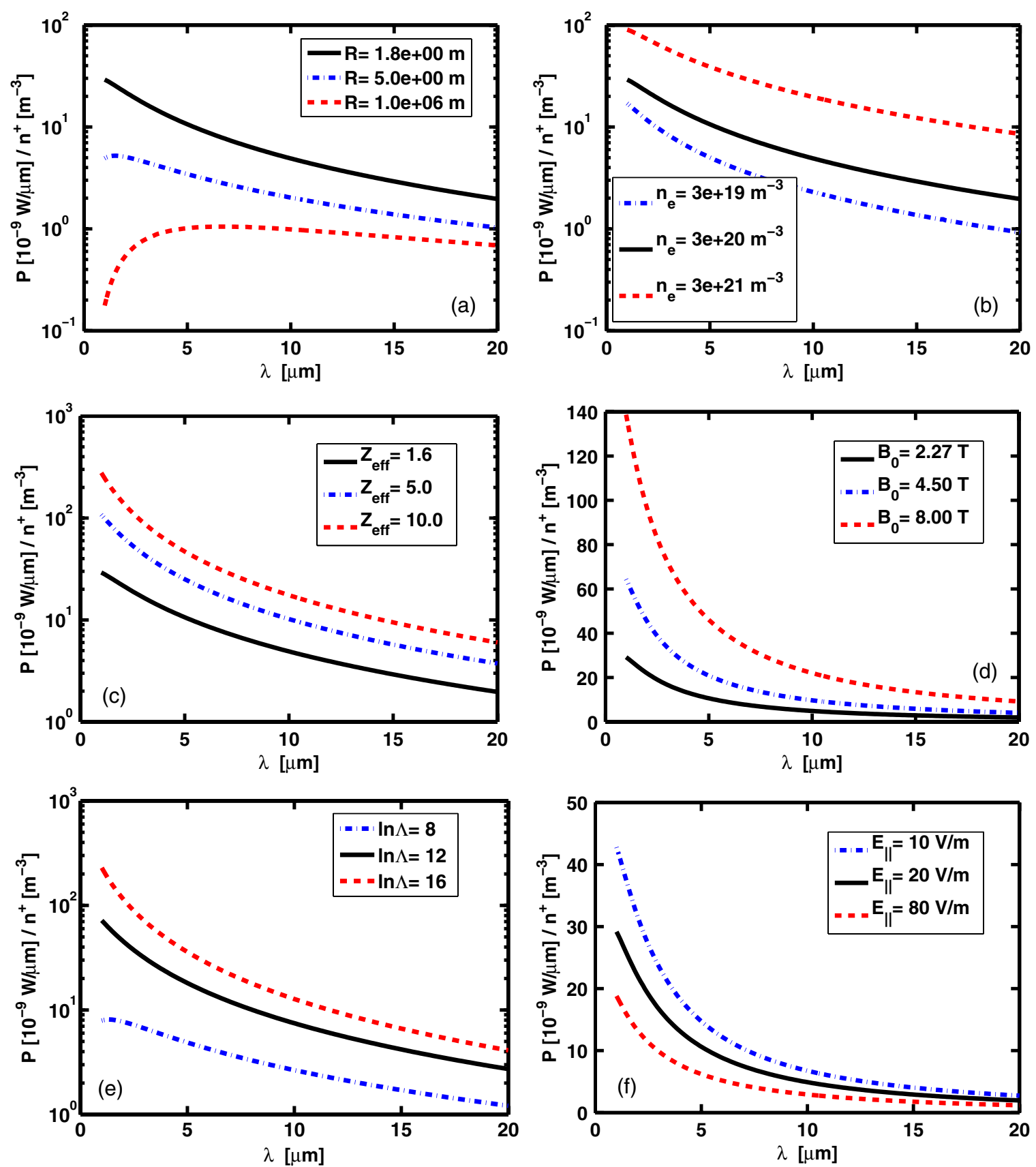

FIG. 4 (color online). Synchrotron radiation spectrum normalized to the positron number density, for an avalanching distribution from (3). Unless otherwise stated, the parameters are the following: magnetic field $B=2.27 \mathrm{~T}$, parallel electric field $E_{\|}=20 \mathrm{~V} / \mathrm{m}$, major radius $R=1.8 \mathrm{~m}$, effective charge $Z_{\text {eff }}=1.6$, Coulomb logarithm $\ln \Lambda=10$, and electron density $n_{e}=3 \times 10^{20} \mathrm{~m}^{-3}$. The rest of the parameters are changed according to the legend of the figures: (a) major radius, (b) density, (c) effective charge, (d) magnetic field, (e) Coulomb logarithm, and (f) parallel electric field.

electric field in the kinetic equation has a surprisingly small effect on the number of tail particles, as has been shown by full numerical simulations of runaway electrons in Ref. [10]. The number of particles in the runaway region can be estimated as $n_{\text {run }}^{+}=4 \pi \int_{p_{c}}^{\infty} f^{+}\left(p^{2}-p_{c}^{2}\right) d p[10]$. Figure 3(b) shows the fraction of positrons that run away as a function of the normalized electric field. Since $p_{c} \ll p_{+}$, almost the whole positron population can be expected to run away $n_{\text {run }}^{+} \simeq n_{+}$.
Positron annihilation has been detected in solar flares [11] and from outside the Solar System [12]. In those cases positron annihilation occurs mostly after positronium formation, which is quite unlikely to occur in tokamaks, where the neutral density is very low. Detection of the annihilation radiation of runaway positrons in tokamaks is difficult, because it is overwhelmed by the bremsstrahlung radiation from the electron population. On the other hand, the synchrotron (and bremsstrahlung) 
radiation of runaway positrons is peaked in the direction opposite from that of the runaway electrons, and this may be possible to detect. The spectral density of the emitted synchrotron radiation for runaway electrons and positrons is approximately [13],

$$
\begin{aligned}
P(\lambda) \simeq & \pi m_{e} c^{3} r_{e} \sqrt{\frac{2 \sqrt{1+\eta^{2}}}{\lambda^{5} R \gamma}}\left[I_{0}(a)+\frac{4 \eta}{1+\eta^{2}} I_{1}(a)\right] \\
& \times \exp (-\xi)[W / \mu \mathrm{m}],
\end{aligned}
$$

where $\lambda$ is the wavelength, $I_{n}(a)$ is the modified Bessel function of order $n, a=(4 \pi / 3)\left(R / \lambda \gamma^{3}\right)\left(\eta /\left(1+\eta^{2}\right)^{3 / 2}\right)$, $\xi=(4 \pi / 3)\left(R / \lambda \gamma^{3}\right)\left(1 / \sqrt{1+\eta^{2}}\right)$ and $\eta=v_{\perp} / v_{d r}$, with $v_{d r}=\gamma v_{\|}^{2} / \omega_{c} R$, and $\omega_{c}$ is the electron cyclotron frequency. The expression (8) is valid for $v_{\|} \gg v_{\perp}, \xi \gg 1$ and $\gamma \gg 1$, which is expected to be satisfied in the case of the runaway positron population. The emission is mainly in the forward direction with an opening angle $\delta=1 / \gamma$. For one positron, with Lorentz factor $\gamma_{+}=10$ and $v_{\perp} / v_{\|}=0.1$, the maximum of the radiation spectrum is around $100 \mu \mathrm{m}$, but the velocity-integrated synchrotron spectrum for a beamlike distribution has a maximum at a lower wavelength, around $1 \mu \mathrm{m}$. Figure 4 shows the synchrotron radiation spectrum and its sensitivity to the major radius, electron density, effective charge, magnetic field, Coulomb logarithm, and parallel electric field. The radiation spectrum is calculated by assuming an avalanching distribution in the form of the runaway electron distribution in Eq. (3), and integrating Eq. (8) in velocity space. The parameters (given in the caption of Fig. 4) are taken from a typical medium-sized tokamak disruption [16]. In most cases, the maximum of the synchrotron radiation spectrum is around $1 \mu \mathrm{m}$, but the absolute magnitude is larger in plasmas with a large number of impurities, high magnetic field, high temperature (through the Coulomb logarithm, see Fig. 4(e)), and lower parallel electric field. The dependence on the parallel electric field may be somewhat counter-intuitive, because more runaways will be produced for high electric fields. However, these runaways will have lower perpendicular momenta, with a corresponding lower synchrotron radiation power. The total radiated power for the parameters used here is only around $0.2 \mathrm{~W}$, but it should be multiplied by $M_{p}$ to account also for positrons produced in collisions with impurities and thermal electrons. Since the radiated power and the spectrum shape are sensitive to the impurity concentration, temperature, and other parameters, detection of positrons should give valuable information about these.

In thunderstorms, positrons that are accelerated by the electric field can produce new runaway electrons via scattering with electrons, which produces secondary avalanches. These secondary avalanches can in turn emit $\mathrm{x}$ rays that Compton scatter or give rise to pair production, resulting in more feedback and more avalanches. It has been shown that the feedback production of runaways by positrons (which were created by the runaway electrons in the first place) is important in lightning initiation $[14,15]$. In tokamak disruptions, the feedback of positrons should be negligible, since the number of positrons is many orders of magnitude lower than the number of runaway electrons.

Conclusion.-Almost all the positrons generated by avalanching runaways will run away and are expected to have lifetimes of several seconds. For an avalanching positron distribution typical of tokamak plasmas, the maximum of the synchrotron radiation spectrum should be around a micron. The radiated power is sensitive to many plasma parameters, especially the number of impurities, temperature, and density. Positron radiation measurements, along with other diagnostics, could become a tool to better understand plasmas containing runaway electrons. These plasmas usually are characterized by sudden cooling and various instabilities, and they are notoriously hard to diagnose. Dedicated measurements of positron radiation may therefore lead to important new insights into the processes that are particular for these plasmas. Although the results of this Letter are mostly relevant for fusion plasmas, the method to determine the source of positrons, their distribution, and the radiation emitted by them can be generalized by changing the parameters and the distribution function of runaways.

This work was funded by the European Communities under the contract of association between Euratom and Vetenskapsrådet. The authors are grateful to P. Jansson, I. Pusztai, and P. Helander for fruitful discussions.

[1] J. A. Wesson et al., Nucl. Fusion 29, 641 (1989).

[2] M. Tavani et al., Phys. Rev. Lett. 106, 018501 (2011).

[3] P. Helander and D. J. Ward, Phys. Rev. Lett. 90, 135004 (2003).

[4] D. A. Gryaznykh, Yad. Fiz. 61, 454 (1998) [Phys. At. Nucl. 61, 394 (1998)].

[5] L.D. Landau and E.M. Lifshitz, Quantum Electrodynamics, Course of Theoretical Physics (Pergamon, New York, 1975), 2nd ed., Vol. 4.

[6] M. N. Rosenbluth and S. V. Putvinski, Nucl. Fusion 37, 1355 (1997).

[7] T. Fülöp, G. Pokol, P. Helander, and M. Lisak, Phys. Plasmas 13, 062506 (2006).

[8] H. Chen et al., Phys. Rev. Lett. 105, 015003 (2010).

[9] D. J. Ward and J.A. Wesson, Nucl. Fusion 32, 1117 (1992).

[10] H. Smith, P. Helander, L.-G. Eriksson, and T. Fülöp, Phys. Plasmas 12, 122505 (2005).

[11] R. J. Murphy, G. H. Share, J. G. Skibo, and B. Kozlovsky, Astrophys. J. Suppl. Ser. 161, 495 (2005).

[12] N. Prantzos et al., Rev. Mod. Phys. 83, 1001 (2011).

[13] R. Jaspers, N. J. Lopes Cardozo, A. J. H. Donne, H. L. M. Widdershoven, and K.H. Finken, Rev. Sci. Instrum. 72, 466 (2001).

[14] J. R. Dwyer, Geophys. Res. Lett. 30, 2055 (2003).

[15] L. P. Babich, E. N. Donskoy, I. M. Kutsyk, and R. A. Roussel-Dupré, Geophys. Res. Lett. 32, L09809 (2005).

[16] G. Papp, M. Drevlak, T. Fülöp, and P. Helander, Nucl. Fusion 51, 043004 (2011). 